Revue d'histoire de l'Amérique française

ZWB REVUE D.HISTOIRE DE L'AMÉRIQUE FRANÇAISE

\title{
Les débuts de l'enseignement de l'histoire et de la géographie au Petit Séminaire de Québec (1765-1830) (suite)
}

\section{Pierre Savard}

Volume 16, numéro 1, juin 1962

URI : https://id.erudit.org/iderudit/302169ar

DOI : https://doi.org/10.7202/302169ar

Aller au sommaire du numéro

Éditeur(s)

Institut d'histoire de l'Amérique française

ISSN

0035-2357 (imprimé)

1492-1383 (numérique)

Découvrir la revue

Citer cet article

Savard, P. (1962). Les débuts de l'enseignement de l'histoire et de la géographie au Petit Séminaire de Québec (1765-1830) (suite). Revue d'histoire de l'Amérique française, 16(1), 43-62. https://doi.org/10.7202/302169ar d'utilisation que vous pouvez consulter en ligne. 


\section{LES DÉBUTS DE L'ENSEIGNEMENT DE L'HISTOIRE ET DE LA GÉOGRAPHIE AU PETIT SÉMINAIRE DE QUÉBEC * $(1765-1830)$ \\ (suite)}

\section{CHAPITRE DEUXIÈME}

L'ABBÉ HOLMES ET SON IMPULSION A L'ENSEIGNEMENT DE L'HISTOIRE ET DE LA GÉOGRAPHIE. L'APPARITION DE L'HISTOIRE NATIONALE

(1830-1845)

L'enseignement de l'histoire et de la géographie connaîtra une période particulièrement brillante sous la longue préfecture de l'abbé Jean ou John Holmes. ${ }^{1}$ Le renouveau des études historiques en France à cette époque et surtout l'action intelligente et vigoureuse du préfet des études sont à l'origine de ce brillant essor.

Le mouvement de renouveau des études historiques amorcé par Chateaubriand au début du XIXe siècle et définitivement mis en branle par les romantiques comme Thierry, Guizot, Thiers, Barante, a bientôt des répercussions dans l'enseignement. En 1827, Michelet publie son célèbre Précis de l'Histoire moderne à l'usage des collèges royaux et de l'École Préparatoire (École Normale) qui, au dire de Camille Jullian, a marqué une «date importante »dans les destinées de l'enseignement et a «chassé ou fait mépriser dans les écoles, ces abominables tableaux chronologiques et synchroniques qui dataient presque tous de l'ancien régime $\gg^{2}$ Dans l'avant-propos, Michelet lui-même déclare que l'enseignement de l'histoire doit présenter «à l'enfant une suite d'images, à l'homme vieux une chaîne d'idées . $^{3}$ Enfin c'est

* Voir notre Revue, XV : 509-525.

${ }^{1}$ L'abbé Holmes est préfet des études au Petit Séminaire de 1831 à 1849 sauf une interruption en 1836-37 pour un voyage en Europe. XXXIII.

2 Camille Jullian, Extraits des historiens français au $X I X^{\bullet}$ siècle, 3 Ibid., XXXIV. 
en 1830 que l'on sépare définitivement l'agrégation d'histoire de celle des Belles-Lettres et qu'ainsi les lycées peuvent se payer le luxe de professeurs spéciaux d'histoire. ${ }^{4}$

Pendant que la vieille Europe romantique assistait à la montée de la vague historique, le Canada français s'apprêtait à franchir une étape brillante dans le perfectionnement de ses institutions scolaires tant au niveau primaire ${ }^{5}$ que secondaire.

L'enseignement secondaire chez nous semble avoir connu avant 1830 une période de stagnation mais peut-être n'était-ce là qu'un retard sur les autres pays et qui n'avait jamais pu être comblé. Certes il faut accepter avec beaucoup de précaution les jugements hâtifs d'Isidore Lebrun, voyageur français, qui déclare en $1833^{6}$ : «Le régime de ces écoles [les collèges classiques et les Petits Séminaires] est donc ecclésiastique, l'enseignement suranné; les méthodes restent stationnaires ... $\gg^{7}$ Le jugement, dégagé de sa forte dose d'anticléricalisme, comporte une part de vérité et un Canadien, Bibaud, Jeune, pourra écrire plus tard: «de la difficulté de trouver des sujets habiles pour remplir les chaires de professeurs dans un si grand nombre d'établissemens (sic), on a dû pendant quelque temps s'apercevoir de la dégénérescence plutôt que du progrès des études ... ${ }^{8}$ Le Petit Séminaire qui n'était pas le moindre des collèges classiques à l'époque ne comptait au dire de Lebrun que 8 professeurs pour plus de deux cents élèves. ${ }^{9}$

4 Georges Weill, Histoire de l'enseignement secondaire en France, 80, et Octave Gréard, Education et instruction. Enseignement secondaire, II: 58 .

5 Abbé Lionel Groulx, L'Enseignement français au Canada, I: 136180 en particulier.

6 L'abbé Groulx le qualifie de «fourrier d'anticléricalisme» op. cit., I: 151. Ses critiques de l'enseignement suscitèrent de vives ripostes comme celle attribuée à l'abbé Ferland et publiée dans le Canadien du 3 et du 5 juillet 1833. M. Marcel Trudel traite de Lebrun dans L'influence de Voltaire au Canada (I: 193s) et souligne que les suggestions de l'auteur relatives à l'enseignement de l'histoire sont empruntées aux Mélanges historiques de Voltaire. Sur Lebrun voir aussi une brève notice dans BRH, XLIV (1938): 183s. 195.

7 Isidore Lebrun, Tableau statistique et politique des Deux Canadas, 8 [Maximillien] Bibaud, Jeund
riels et intellectuels du Canada, 23.

9 Lebrun, op. cit., 196. 
Enfin arrive l'abbé Holmes et il s'ensuit une véritable révolution dans l'enseignement, d'abord au Petit Séminaire mais qui s'étend bientôt dans les autres établissements. Nous n'avons pas l'intention de retracer ici en détail la carrière étonnante de cet éducateur célèbre. ${ }^{10}$ Né dans le Vermont de parents protestants, il songe dès son jeune âge à se faire ministre de son Église. Sur le refus de son père il s'enfuit de la maison et vient chercher asile dans la province de Québec. A la suite d'une série de circonstances qui relèvent presque du roman, il apprend le métier d'instituteur chez le célèbre maître Burrough des Trois-Rivières, se convertit au catholicisme et enfin est ordonné prêtre le 5 août 1823. Il déploiera son zèle de missionnaire dans les Cantons de l'Est pendant quelques années jusqu'à ce que sa santé fragile le force à songer à des occupations d'un autre genre.

En 1828, après avoir hésité entre les « Français » de Montréal et les «Canadiens » de Québec, l'abbé Holmes décide d'entrer au Petit Séminaire de Québec où l'on demandait alors un professeur de philosophie. L'année suivante, il devient un des directeurs de l'établissement. Et en 1831, on le nomme préfet des études.

Travailleur acharné, esprit ouvert, intellectuel fervent, il s'adonna à tant de recherches et assimila tant de notions qu'il en vint à enseigner avec la même facilité le latin, le grec, la langue hébraïque, l'astronomie, la géographie, l'histoire naturelle, sans compter la poussée qu'il donna à la musique, au dessin et à l'art oratoire ... écrit un biographe. ${ }^{11}$

L'annaliste des Ursulines pouvait écrire avec raison que l'abbé Holmes avait donné à l'éducation de son temps «un élan

${ }^{10}$ La carrière de l'abbé Holmes a été racontée sous une forme légèrement romancée par Mgr Maurice O'Bready, John ou Jean Holmes. Un extrait de ce travail, intitulé Un pédagogue dynamique, a été publié dans le Devoir de 14 mars 1957, p. 24. Sur l'abbé Holmes on ajoutera à la bibliographie de Mgr O'Bready un article de M. Mason Wade dans Culture, XV (1954) : 3-16, intitulé The Contribution of abbé John Holmes to Education in the Province of Quebec. Même après ces travaux, il reste encore bien des questions à se poser au sujet du célèbre éducateur. Où par exemple a-t-il pris son goût extraordinaire pour la géographie ? Dans quelle mesure ses méthodes innovatrices s'inspirent-elles de son ancienne mère-patrie?

11 O'Bready, op. cit., 142s. 
prodigieux ».12 Et Morgan dira plus tard que l'abbé Holmes a inauguré «the dawn of a new era in the history of college ». ${ }^{13}$

L'histoire et la géographie occupaient une place de choix dans les préoccupations intellectuelles et pédagogiques de l'abbé Holmes. Il développa en particulier certaines techniques pour rendre plus profitable l'étude de ces deux disciplines. C'est ainsi qu'il renouvela la pratique des examens publics, introduisit l'usage des cahiers d'histoire chez les élèves et fit même donner une partie de l'enseignement de l'histoire et de la géographie en anglais. C'était la coutume, héritée des Collèges des Jésuites, de tenir dans les institutions secondaires de jadis des exercices ou examens publics à la fin de l'année. Le Collège de Québec avait eu les siens. En 1775 et en 1790 , le Petit Séminaire fera même imprimer des prospectus invitant parents et amis à de telles séances. ${ }^{14}$ Mais il fut donné à l'abbé Holmes de porter ce procédé à un point de perfectionnement inconnu jusque-là.

En 1830, écrit encore l'annaliste des Ursulines, il établissait au collège de cette ville un examen de trois jours, où les élèves étaient questionnés par des personnes de l'auditoire sur toutes les branches de l'instruction... On conçoit que ce mode était des plus avantageux à la cause de l'Education, à une époque où il fallait éveiller les esprits. Les examens sous cette forme [après 1853, ils devinrent semi-publics et durèrent huit jours] subsistèrent pendant plus de vingt ans. ${ }^{15}$

Le Canadien de l'époque nous a laissé des descriptions enthousiastes de ces séances. Le compte rendu de 1833, attribué à Étienne Parent, salue l'ouverture d'une «ère nouvelle pour l'instruction classique » et célèbre un «plan d'enseignement qui, comme autrefois, ne s'adresse plus simplement à la mémoire sans appeler l'exercice d'une autre faculté bien plus précieuse

12 [Adèle Cimon], Les Ursulines de Québec depuis leur établissement jusqu'à nos jours, III : 670 .

13 Henry J. Morgan, Sketches of Celebrated Canadians and persons connected with Canada, from the earliest period in the history of the province down to the present time, 281.

14 Groulx, op. cit., I: 114.

15 [Adèle Cimon], op. cit., IV : 732. 
encore, le jugement ». Et l'auteur de louer hautement l'« ingénieux Préfet des Etudes 》 grand responsable de ce succès. ${ }^{16}$

A la vérité, les méthodes de l'abbé Holmes ne manquaient ni d'intérêt ni d'efficacité. C'est ainsi que le brillant pédagogue avait introduit dans ces séances publiques la dramatisation de la géographie. On sait que le début du XIXe siècle vit un nombre considérable de ces expéditions destinées à faire connaître l'intérieur des continents. Déjà en 1778, l'African Association entreprenait l'exploration moderne du Soudan. Et le Français Caillé s'enfonce à quatre reprises entre 1816 et 1827 à l'intérieur du continent noir. C'est aussi de cette époque que datent les fameuses expéditions de l'allemand Humboldt en Amérique du Sud. A partir de ces faits qui défrayent la chronique du temps le préfet des études décide de rajeunir l'enseignement de la géographie. En 1833 par exemple, un groupe d'élèves de sixième représentent «une commission d'explorateurs 》 et ils doivent, «après leurs excursions dans les différentes parties du pays [le Canada], rendre compte de ce qu'ils ont vu devant une société savante... ». ${ }^{17}$ L'année suivante le prospectus des examens publics annonce de

Nouveaux détails sur la géographie de l'Amérique Britannique - Rapport de plusieurs jeunes voyageurs - Exhibition d'un grand nombre d'objets d'histoire nationale, de costumes sauvages, etc. Discours d'un sauvage du N.O., etc.... ${ }^{18}$

Procédé analogue à la séance publique de 1835, célébrée en ces termes par le journaliste du Canadien:

Nous avons déjà eu l'occasion dans une brève notice précédente d'applaudir à l'heureuse idée des professeurs de dramatiser l'étude de la géographie, étude assez sèche et ingrate de sa nature: telle elle était au moins de notre temps. Mais faites partir vos jeunes géographes de Québec, et faites leur faire le voyage du Mexique ou des Etats-Unis; faites leur

${ }^{16}$ Le Canadien, 16 août 1833.

17 Loc. cit.

18 Le Canadien, 3 octobre 1834; voir aussi le programme des Examens publics du Séminaire de Québec [...] en 1834. 
remarquer sur leur route tout ce que la nature, l'art et la société offrent de plus remarquable, selon leur âge et le degré de culture de leur esprit; - cette étude hérissée de mots devient un voyage pittoresque et amusant, et des enfans de sixième dévoreront leurs cahiers, qu'ils auraient autrement repoussés de dégoût. ${ }^{19}$

Cette étude portait un titre suggestif : «Géographie amusante ». En 1839 c'est l'Asie qui prend la vedette:

Dans le « Rapport des jeunes Voyageurs à leur retour de diverses contrées de l'Asie », on voyait ces jeunes rapporteurs sous différents costumes Asiatiques anciens et modernes, aussi fidèles que possible. Par exemple on avait le grand Lama, le grand Turc et l'Aga des Janissaires, un Mufti, un Chevalier de Malte, un Chinois, un Cosaque, deux Pellerins (sic) Grecs etc.

«Cela plaît et instruit en même temps » ajoute le journaliste. ${ }^{20}$

Les cahiers d'histoire constituent une des innovations les plus fécondes de l'abbé Holmes. Il s'agit ici de travaux d'élèves et non de cahiers de notes de cours professés $e x$ cathedra comme nous en avons retrouvé plusieurs au cours du XIXe siècle. Le récit du Canadien de 1833 déjà cité nous a laissé un éloge enthousiaste de la pratique au plus haut point profitable de cette technique. Les élèves de cinquième, de quatrième et de troisième ont été invités, cette année-là à

écrire eux-mêmes chacun son cahier, non pas sous la dictée du maître, mais d'après ses lectures et ses recherches, le maître n'ayant fait que les diriger dans leurs études. Puis, ajoute le compte rendu de la séance, les écoliers ont été interrogés, chaque classe sur l'histoire qu'on lui avait donnée à écrire, et chacun a répondu d'après son propre cahier écrit et digéré (sic) par lui-même ... ${ }^{21}$

Pour la documentation, plusieurs se sont rendus à la Bibliothèque de la Chambre d'Assemblée, au dire du journaliste qui par-

\footnotetext{
19 Le Canadien, 14 août 1835.

20 Le Canadien, 14 août 1839.

${ }^{21}$ Le Canadien, 16 août 1833.
} 
le en connaissance de cause. ${ }^{22}$ Et les cahiers d'histoire circulent parmi les invités durant la séance.

Pendant que ces Messieurs répondaient aux questions qu'on leur faisait, leurs cahiers ont été passés de main en main dans la salle, et nous en avons parcouru un sur l'Histoire de France qui nous a rempli d'étonnement par le jugement et le soin avec lequel il a été rédigé.

Et le chroniqueur de conclure justement: « Ce travail outre l'utile exercice qu'il donne à l'esprit des élèves, est aussi bien propre à donner aux précepteurs la clé de l'intelligence de chacun des écoliers, chose très essentielle.» ${ }^{23}$ Il ne nous est malheureusement resté aucune trace de ces cahiers d'histoire.

Nous avons mentionné plus haut l'impulsion donnée par l'abbé Holmes à l'étude de la langue anglaise. Pour comprendre le sens de cette démarche il faut non seulement penser à l'origine du préfet des Études mais surtout au fait que Québec comptait en 1830 une forte proportion d'habitants d'expression anglaise. ${ }^{24}$ Le célèbre Tocqueville écrira vers cette époque:

Les quatre cent mille Français du Bas-Canada forment aujourd'hui comme les débris d'un peuple ancien perdu au milieu des flots d'une nation nouvelle. Autour d'eux la population étrangère grandit sans cesse; elle s'étend de tous côtés; elle pénètre jusque dans les rangs des anciens maîtres du sol, domine dans leurs villes... ${ }^{25}$

En 1839, l'abbé Holmes introduit l'étude de la géographie en anglais en utilisant comme manuel le Catechism of Geography de Pinnock. ${ }^{26}$ L'année suivante, apparaît au programme d'étude l'«Ancient History», la «Roman History» et l'«History of

22 En effet, l'auteur présumé de ce compte rendu, Etienne Parent, administre de 1832 à 1835 la Bibliothèque de la Chambre d'Assemblée. Sur ce point, voir Aegidius Fauteux, Les bibliothèques canadiennes, 31s.

${ }^{23}$ Le Canadien, 16 août 1833.

$24 \mathrm{M}$. Raoul Blanchard, dans son esquisse géographique de Québec nous apprend qu'en 1830 le tiers de la population québecoise est d'expression anglaise. (L'Est du Canada français, II: 208s.).

25 Alexis de Tocqueville, De la démocratie en Amérique, I: 426.

${ }^{26}$ Le Canadien, 14 août 1839. 


\section{NOUVEL ẠBRÉGÉ}

$\mathbf{D E}$

\section{GÉOGRAPHIE MODERNE,}

SUIVI D'UN PETIT ABRÉGE

$\mathbf{D E}$

GÉOGRAPHIE ANCIENNE,

A l'Usage de la Jeunesse.

\section{QUÉBFC :}

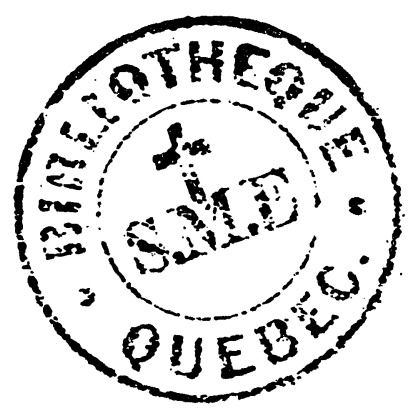

Se troúve chez Neruson \& Cowan, N 0 . 3, Côte de la Basse.Vill.

$$
1831 .
$$

Page-titre de la première édition de l'Abrégé de l'abbé Holmes. Opuscule de 63 pages publié sans nom d'auteur. 
England.$^{27}$ En 1841, on accorde «un prix de Géographie anglaise en Septième et en Huitième $\gg .{ }^{28}$ Plus tard on enseignera aussi l'histoire moderne en anglais. ${ }^{29}$ L'enseignement de l'histoire et de la géographie en anglais disparaîtra après le départ de l'abbé Holmes.

Il semble que les manuels alors employés pour cet enseignement aient à peu près tous fait partie de la collection Pinnock d'origine anglaise mais également en vogue et souvent rééditée aux Etats-Unis. La Bibliothèque de l'Université Laval possède une trentaine de ces volumes dont elle semble avoir hérité pour la plupart du Petit Séminaire. Ces manuels sont des abrégés de géographie, d'histoire ancienne, moderne, d'histoire de la France, de l'Angleterre, de l'Amérique, tous publiés en anglais et la plupart avant 1850. Les premiers manuels de cette collection fameuse avaient eu pour auteur nul autre que le grand écrivain anglais Oliver Goldsmith.

La partie la plus durable et la mieux connue de l'œuvre pédagogique de l'abbé Holmes reste sans contredit la publication de son Abrégé de géographie moderne justement célèbre et qui sera en usage dans les collèges classiques durant près de troisquarts de siècle. L'ouvrage qui connut au moins neuf éditions ${ }^{30}$ fut traduit en allemand, en anglais et aurait même été adopté comme manuel dans certains collèges américains. ${ }^{31}$ $1839-40$.

27 Programme abrégé du cours d'étude du Petit Séminaire pour l'année

28 Le Canadien, 16 août 1841.

29 Catalogue des officiers et élèves du Séminaire de Québec - 1847$1848-, 18$.

30 Une liste des éditions du manuel de Holmes a été publiée dans $\mathrm{BRH}$, LIII (1947): 160s. Les premières éditions ne portent pas de nom d'auteur. En 1831 paraît la première partie de l'Abrégé qui comprend les notions préliminaires et la géographie de l'Amérique (XII-53 pages). En mai 1832, réédition du même texte auquel on a ajouté la géographie de l'Europe (XII-160 pages). Enfin en août 1833 la 《Seconde partie», paginée de 161 à 277 avec en plus un appendice de XXX pages et un abrégé de géographie sacrée de 16 pages, complète la publication.

31 Il n'est pas sans intérêt de faire remarquer ici qu'après 1830, aux Etats-Unis, l'enseignement de la géographie connaît un regain considérable inspiré en cela par l'exemple allemand et stimulé par des savants comme William Woodbridge, auteur d'atlas célèbres dont la Bibliothèque du Séminaire du temps possède plusieurs éditions. Sur ce point consulter Walter S. Monroe, éd., Encyclopedia of Educational Research, à l'article: Social studies. 
Les contemporains ne tarissent pas d'éloges sur la qualité de cet ouvrage. Le bibliophile Faribault le loue hautement en $1837^{32}$ et Bibaud qui a bien connu l'Abrégé le qualifie de «petit chef-d'œuvre en ce genre $\gg^{33}$ On peut aisément se faire une idée de la valeur du manuel de Holmes en le comparant avec un ouvrage paru quelques années auparavant sur le même sujet. ${ }^{34}$ Le manuel de Holmes compte plus de 335 pages, l'autre, une cinquantaine. Et surtout l'ouvrage du préfet des études du Petit Séminaire l'emporte nettement sur le plan pédagogique et scientifique.

Il n'est pas inutile de s'arrêter un moment sur le contenu de l'Abrégé de géographie moderne dont la publication marque une date décisive dans l'histoire de la pénétration de la géographie et son enseignement au Canada français.

Bien que l'auteur cite très rarement ses sources, il semble s'inspirer des travaux les plus récents et les plus sûrs de son temps, tels ceux de Malte-Brun qui fait autorité à l'époque. ${ }^{35}$ Notons chez l'auteur de l'Abrégé le souci de mentionner les toutes récentes découvertes, ${ }^{36}$ d'apporter les statistiques les plus à jour possible, ${ }^{37}$ d'indiquer les derniers changements politiques..$^{38}$ Et quand l'information fait défaut, l'auteur l'avoue honnêtement:

${ }^{32}$ G. B. Faribault, Catalogue d'Ouvrages sur l'Histoire de l'Amérique $[\ldots] 63$.

33 Bibaud, op. cit., 45. en 1828

34 Géographie élémentaire à l'usage du Séminaire de Nicolet publiée

35 [Abbé Jean Holmes], Abrégé de géographie moderne [...], 222. 36 Ibid., 229: "La découverte toute récente des embouchures du Niger dont une expédition anglaise explore actuellement (Mai, 1833) le cours, sera sans doute une époque bien importante dans cette partie du monde. 》 37 Ibid., 106: «La population de Paris en novembre 1831 était de 770,000 habitants. \L'édition de cette partie de l'Abrégé est de mai 1832 ...

38 Ibid., 157: "Les Grecs secouèrent le joug des Tures en 1820. La lutte opiniâtre et sanglante qu'ils eurent à soutenir, ne s'est terminée en 1829, que par l'intervention de l'Angleterre, de la France et de la Russie. A cette époque, leur indépendance fut admise par la Porte et par les autres états européens. Mais cette nation malheureuse paraît encore en proie à de grandes dissentions politiques... traité d'Andrinople qui, en septembre 1829, consacrait l'indépendance de la Grèce. 
Les divisions civiles de la Turquie sont trop arbitraires et trop peu connues pour qu'il soit possible de les indiquer d'une manière satisfaisante... ${ }^{39}$

Malgré sa rigueur scientifique l'exposé ne laisse pas d'être intéressant voire amusant. Les étudiants apprennent ainsi

qu'il n'existe en Irlande ni serpens (sic), ni vipères, ni taupes, ni crapauds, et que les grenouilles y ont été importées de l'Angleterre. ${ }^{40}$

Tout intéresse le géographe, non seulement le pittoresque, ${ }^{41}$ mais aussi l'utile. Dans le chapitre consacré aux Îles Britanniques, on étudie successivement le gouvernement (attributions du Roi, composition et fonction de la Chambre des Lords), la population en général (religion, effectifs militaires, population de l'Empire). Puis vient l'étude des diverses parties composantes des Îles Britanniques: Angleterre, Pays de Galles et Irlande. De l'Angleterre, les élèves étudient les bornes territoriales, les divisions, la configuration du sol, les cours d'eau, les lacs, les canaux et les chemins de fer, grande nouveauté de l'époque. ${ }^{42}$ Suivent des considérations sur le réseau routier, le climat, les productions (culture et élevage), les mines (et eaux minérales), les manufactures et le commerce. La capitale, Londres, reçoit une attention spéciale. L'auteur parle de ses édifices remarquables, de ses églises, de ses ports, des établissements scientifiques et de bienfaisance qu'on y trouve. Après un mot sur chacune des autres villes d'importance, le chapitre se termine par des considérations sur l'éducation, la population, les antiquités romaines et les îles qui dépendent de l'Angleterre. Ce plan se retrouve mutatis mutandis pour chaque pays étudié.

Au chapitre de la valeur scientifique du manuel, il faut signaler son Appendice remarquable. On trouve là rassemblées des données fort précieuses. D'abord un extrait du célèbre traité

39 Ibid., 153.

40 Ibid., 76.

41 Ibid., 134. On y trouve une description des « Cinganes ou Zigennes » propre à piquer la curiosité.

42 Ibid., 64: "Des chemins de fer (rail-road) ont été construits dans plusieurs endroits; le plus beau et le plus important est celui entre Liverpool et Manchester de la longueur de 37 milles. » 
anglo-russe de 1825 intitulé : «Limites de l'Amérique britannique et de l'Amérique russe », « pièce très peu connue », souligne Faribault. ${ }^{43}$ Cet appendice comprend aussi entre autres un «tableau statistique du Bas-Canada d'après le recensement de 1831 » et un tableau statistique des Etats-Unis (population par état en $1790,1800,1810,1820,1830$, esclaves par état, statistiques religieuses, etc....). Autant de renseignements fort utiles et auxquels l'accès restait souvent difficile.

Les qualités pédagogiques du manuel ne le cèdent en rien à celles qu'il possède sur le plan scientifique. Ordonnance claire, textes courts et adaptés à l'esprit des élèves, abondance de traits propres à susciter l'intérêt, autant de moyens de rendre l'étude à la fois profitable et plus facile. Une qualité en particulier mérite d'être soulignée ici: son caractère proprement canadien. Jusque-là (pour ne pas parler des manuels européens où la part du Canada restait négligeable) les manuels imprimés au Canada ne faisaient pas grand cas de notre pays. ${ }^{44}$ L'Abrégé de géographie moderne accorde enfin à l'Amérique l'attention qu'elle mérite. L'étude de notre continent vient en premier lieu dans le manuel et on décèle tout au cours de l'Abrégé une tendance à procéder du connu des élèves vers l'inconnu, en même temps qu'un effort pour libérer l'étude de la géographie d'une foule de singularités exotiques souvent inutiles sinon invraisemblables.

Ajoutons que l'abbé Holmes a très bien compris le rôle primordial des cartes dans l'enseignement de la géographie. Bien que ses manuels n'en contiennent aucune, ceci sans doute dû à des problèmes d'impression, l'auteur déclare nettement que les professeurs qui utilisent son manuel

sont priés d'observer que les élèves en les récitant, doivent avoir sous les yeux les cartes des différents pays de la terre, au moins des cartes générales de l'Amérique, de l'Europe, de l'Asie, de l'Afrique, et de l'Océanie. ${ }^{45}$

\footnotetext{
43 Op. cit., 63.

44 On n'a qu'c̀ comparer sur ce point l'Abrégé de Holmes avec la Géographie de 1804 et celle du collège de Nicolet déjà citées.

45 [Holmes], op. cit., Avertissement (de l'édition de 1831).
} 
A part la publication de son Abrégé, l'abbé Holmes encouragea les études géographiques au Petit Séminaire en enrichissant la Bibliothèque d'une foule d'ouvrages européens et américains de première valeur. Ainsi c'est sans doute lui qui fit acheter les éditions de la Géographie de Malte-Brun de 1830 et de 1831 et l'atlas correspondant au premier de ces ouvrages. Nous avons relevé une vingtaine d'autres traités et atlas de première valeur acquis suivant toute vraisemblance durant le 1952 fera apparaître les concordances : ${ }^{47}$

Avec l'abbé Holmes la géographie atteint dans les programmes le point de fixation qu'elle a gardé sensiblement jusqu'à nos jours. Une simple comparaison des programmes de 1850 et de 1952 fera apparaître les concordances : ${ }^{47}$

1850

1952

Classe préparatoire Notions préliminaires

Septième

id.

Sixième

L'Europe

Notions générales

(Éléments latins)

Cinquième

(Syntaxe)

L'Asie

Afrique,

Asie, Océanie

Quatrième

Afrique et

Océanie

Europe

(Méthode)

Troisième

(Versification)

Amérique Amérique

Après le départ de l'abbé Holmes, la géographie restera en vogue au Petit Séminaire comme en témoignent de nombreux articles d'intérêt géographique parus dans l'Abeille. La seule

46 Tels les traités de Woodbridge (Hartford, éditions de 1823 et de 1830), Arrowsmith (Londres, 1835), etc.

47 Catalogue des officiers et des élèves du Séminaire de Québec, 185051, 28ss. Université Laval, Faculté des Arts. Cours classique des garçons. Règlement de l'Immatriculation et de Baccalauréat. Programme des Etudes, No 20, 51-56. 
année 1853-54 voit des articles sur le commerce de l'Angleterre, ${ }^{48}$ sur le Nagpour, «nouveau territoire que les Anglais viennent d'annexer à leur vaste empire des Indes $\gg,{ }^{49}$ sur la population de la Chine d'après « un nouvel ouvrage publié sur le pays ${ }^{50}$ sur celle de l'empire turc, ${ }^{51}$ etc. Les membres de la Société Laval s'intéressent également à la géographie. A la séance du 7 avril 1853, un élève «démontre quel intérêt nous devons porter à l'étude de la position géographique des pays, de leur climat, de leurs productions, et de leur commerce, à l'étude du gouvernement et de la religion de ceux qui les habitent... $\gg^{52}$ A la séance du 14 avril suivant, les membres entendent un exposé sur la Nouvelle-Zélande. ${ }^{53}$

Cet intérêt pour la géographie après le départ de l'abbé Holmes est sans doute largement redevable à l'action de l'abbé L.-O. Gauthier, disciple du grand pédagogue dont il rééditera l'Abrégé à plusieurs reprises. Parallèlement à son action dans l'enseignement de la géographie l'abbé Holmes exerça une activité considérable sur la pédagogie historique de son temps. D'ailleurs l'histoire occupait une place de choix dans les préoccupations intellectuelles de l'abbé et sa culture historique passait pour remarquable. Ainsi l'auteur utilisera largement ses connaissances historiques dans ses Conférences de Notre-Dame de Québec qui l'ont rendu célèbre. Dès sa première conférence l'auteur déclare explicitement:

Le fond sera puisé dans l'histoire ... Je m'appuierai au besoin sur la science et les savants; j'interrogerai la nature, les monuments et les arts, les langues et les coutumes des peuples...54

C'est à l'abbé Holmes que l'on doit l'introduction de l'histoire moderne dans les programmes à la place des histoires nationales

48 L'Abeille, 27 octobre 1853.

49 Ibid., 30 mars 1854.

50 Ibid., 17 novembre 1853.

51 Ibid., 24 novembre 1853.

52 Ibid., 3 et 10 mai 1853.

53 Ibid., 10 et 19 mai 1853.

54 Abbé Jean Holmes, Conférences de Notre-Dame de Québec, 40. 
de France et d'Angleterre.55 Il s'occupa aussi activement de l'édition de manuels d'histoire.

Vers 1831, nous raconte l'annaliste des Ursulines, l'abbé Holmes... faisait imprimer l'Histoire $A n$ cienne, l'Histoire Romaine, l'Arithmétique de Bouthilier, la Grammaire française de Lhomond. ${ }^{56}$

On trouve encore aujourd'hui dans les vieilles bibliothèques des exemplaires des deux premiers de ces ouvrages. Tout porte à croire qu'ils reproduisent à peu près intégralement et sans nom d'auteur des ouvrages publiés en France peu auparavant.57 Une légende tenace lancée par on ne sait qui au juste fait de l'abbé Holmes l'auteur de ces ouvrages qu'il s'est contenté d'éditer. ${ }^{58} \mathrm{Il}$ y a peu à dire sur le contenu de ces manuels dont les auteurs s'en tiennent à Rollin et ses continuateurs Lebeau et Crevier. ${ }^{59}$ L'abbé Holmes aurait composé une Histoire Moderne dont L.-M. Darveau disait «nous n'avons jamais rien lu d'aussi bien raconté dans ce genre ${ }^{60}$ Il ne reste aucune trace de ce travail qui n'était peut-être que le manuscrit de son cours donné au Petit Séminaire

Il semble qu'on doive attribuer à l'initiative du préfet des études du Petit Séminaire la publication à Québec en 1837 d'un Nouvel abrégé de l'histoire de la France depuis Pharamond jus-

55 Programme abrégé du Cours d'étude du Petit Séminaire de Québec pour l'année 1840-41.

56 [Adèle Cimon], op. cit., IV: 670, note 1.

57 Le fait semble évident pour l'Histoire romaine qui reproduit mot à mot ou presque une Histoire romaine depuis la fondation de Rome jusqu'a la fin de l'Empire d'occident [... ] publiée à Lyon. (Nous n'avons retrouvé de ce dernier ouvrage qu'une édition de 1832 qualifiée de «nouvelle ».) Pour l'Histoire ancienne on peut déduire de même car l'Avertissement des éditeurs de l'Histoire romaine mentionne une Histoire sainte et une Histoire ancienne «sur le même plan et par le même auteur» déjà publiées à Québec.

58 Le plus récent biographe de l'abbé Holmes répète cette légende: O'Bready, op. cit., 145. Par contre l'annaliste déjà citée dit bien clairement «fait imprimer » et l'abbé Taschereau, disciple de l'abbé Holmes, dans la précieuse notice nécrologique qu'il a composée à la mort de son maître, ne mentionne parmi les ouvres de ce dernier que «les trois éditions du traité de géographie », L'Abeille, 23 juin 1852.

59 Voir par exemple l'«Avis de l'auteur» dans l'Histoire romaine, éditée en 1831, 6.

60 L.-M. Darveau, Nos hommes de lettres, 272. 
qu'à nos jours. Il s'agit d'une histoire assez sèche divisée par règnes. De temps à autre on a ajouté aux chapitres quelques renseignements sur les «événemens (sic) mémorables, personnages célèbres, inventions, découvertes, érections de monumens (sic) ...» L'ouvrage publié en France sous la Restauration est bien entendu réactionnaire et prend parti violemment contre la Révolution française

principalement causée par les écrits d'une foule d'hommes, dont quelques-uns voulaient le bien de la nation, mais dont le plus grand nombre ne cherchaient qu'à parvenir à la fortune et aux honneurs en faisant croire au peuple qu'il travaillait pour lui. ${ }^{61}$

$\mathrm{Au}$ chapitre des morts célèbres, après celles de Voltaire et du bailli de Suffren, on signale en 1793 celle de «Lamoignon de Malesherbes le vertueux défenseur de Louis XVI. Il fut moissonné par la faux révolutionnaire ${ }^{62}$ Enfin on termine par l'apothéose du roi Charles $X$ sous le règne duquel «jamais la France n'avait joui d'autant de bonheur et de prospérité ... ${ }^{\mathbf{6 3}}$ C'est à la même époque qu'on publie également à Québec l'Abrégé de Mythologie de Moustalon qui sera réédité en 1849. L'avertissement annonce que c'est « peut-être le meilleur et le plus satisfaisant de tous ceux qu'on a jusqu'à présent rédigés en français pour l'usage de la jeunesse $\gg^{64}$ Dans l'introduction, l'auteur tout en reconnaissant l'immoralisme et l'invraisemblance des récits fabuleux insiste sur l'importance de leur étude car la mythologie reste

le ressort principal et quelquefois même la base des plus beaux ouvrages en vers, tant anciens que modernes, que d'ailleurs la peinture et la sculpture lui doivent en partie leurs chefs -d'œuvre. ${ }^{65}$

Toute cette activité qui peut être attribuée principalement au dynamique préfet des études ne doit pas nous faire oublier les

61 [Anonyme], Nouvel abrégé de l'histoire de la France depuis Pharamond jusqu'à nos jours, 74 .

62 Ibid., 76.

63 Ibid., 81.

64 M. Moustalon. Abrégé de Mythologie à l'usage des maisons d'éducation, Avertissement.

65 Ibid., 2s. 
autres éducateurs du temps qui semblent avoir partagé jusqu'à un certain point l'intérêt de l'abbé Holmes pour l'histoire et la géographie. Tel l'abbé Jérôme Demers dont la riche bibliothèque renfermait des ouvrages historiques intéressants. ${ }^{66}$

Enfin, il y aurait beaucoup à dire au sujet de l'influence incalculable de l'abbé Holmes. Qu'il suffise de rappeler que l'abbé Taschereau (le futur cardinal) et l'abbé Laverdière doivent sans doute à ce pédagogue remarquable leur goût pour l'histoire. ${ }^{67}$ C'est au temps de l'abbé Holmes que fut introduit dans les programmes d'étude l'enseignement de l'histoire du Canada. Pour comprendre l'apparition de cette nouvelle matière, il faut dire un mot sur les conditions faites jusque-là à l'histoire nationale et sa pédagogie tant en France que chez nous.

L'enseignement de l'histoire nationale en France dans les établissements secondaires remonte à très loin. On connaît un manuel employé à cet usage qui date de $1653 .^{68}$ Mais l'attention démesurée accordée pendant longtemps à l'histoire ancienne et romaine ne put que paralyser le développement de cet enseignement, d'où sa place encore si réduite dans les programmes au début du $\mathrm{XIX}^{\mathrm{e}}$ siècle.

Or le début et la première moitié du siècle voient précisément le triomphe du «romantisme rétrospectif » et le déchâ̂nement des nationalismes. Et c'est dans l'histoire nationale que les intellectuels d'alors vont chercher leurs principes et leurs raisons de lutter. En quelques années l'histoire nationale prend une importance capitale et sa situation dans l'enseignement

66 L'abbé Demers lèguera sa bibliothèque au Petit Séminaire. Nous avons retrouvé quelques volumes portant son ex-libris à la Bibliothèque de l'Université Laval. En particulier, l'abbé Demers possédait un exemplaire des célèbres Leçons d'histoire, prononcées à l'école normale, en l'an III de la République française $[\ldots]$ de C.-F. Volney. Le propriétaire y a inscrit: «Ce livre est très mauvais.» Nous avons aussi retracé une traduction de Herder, un des historiens allemands dont l'influence sera la plus considérable au siècle dernier sur la philosophie de l'histoire.

67 Tous les deux seront professeurs d'histoire au Petit Séminaire. L'abbé Taschereau a laissé une volumineuse histoire du Séminaire (plus de 1200 pages) restée manuscrite. Nous verrons plus loin l'action de l'abbé Laverdière.

68 Marcel Reinhard, Histoire des Histoires de France dans Histoire de France [...] I: 10 . 
change du tout au tout. Ainsi en 1852, les programmes français accordent la première place à l'histoire nationale. ${ }^{69}$ Il faut dire qu'entre-temps, il y a eu les travaux de Thierry, de Michelet, de Thiers et de Guizot. La science historique est alors devenue une «institution nationale » pour employer le mot de l'un d'eux. ${ }^{70}$ Avant 1830, les Canadiens semblent avoir manifesté bien peu d'intérêt pour leur propre histoire. Certes, en 1815 Smith avait publié son History of Canada ${ }^{70 a}$ et John MacGregor dans son British America traitait largement de l'histoire de notre pays. Mais ces œuvres écrites à la louange de la métropole semblent avoir peu intéressé les Canadiens français. Bibaud, Jeune pourra écrire plus tard que «les Canadiens s'étaient montrés en général peu curieux de leur passé surtout avant la création de la Société Littéraire et Historique. ${ }^{71}$ Après 1830 , l'intérêt pour l'histoire nationale se concrétisera dans les travaux de Bibaud et dans les manuels scolaires de Perrault en attendant la synthèse brillante de Garneau.

C'est au cours primaire que l'enseignement de l'histoire du Canada fait d'abord son apparition. ${ }^{72}$ La première expérience connue est celle du docteur Labrie qui soutenait une école à StEustache, école dont il avait lui-même élaboré le programme d'étude. Aux examens de 1826, les élèves furent questionnés sur l'histoire et en particulier l'histoire du Canada «pendant une heure $\gg^{73}$. L'enseignement de l'histoire nationale se répandra peu à peu dans les écoles primaires mais ne s'imposera de façon définitive que longtemps plus tard après les travaux de Garneau. Au cours secondaire, on semble éprouver plus de réticence. Certes il était question de notre pays à l'occasion de l'histoire de France ou d'Angleterre mais on peut difficilement parler ici d'un enseignement de l'histoire nationale. Jusqu'à

69 Gréard, op. cit., II : 79.

70 Thierry cité dans Jullian, op. cit., XLI.

70a Livrée au public quelques années plus tard. en 1824.

71 Bibaud, op. cit., 41. La Société Littéraire et Historique fut fondée

72 C.-J. Magnan, «L'histoire du Canada à l'école primaire 1825-1925 》, dans Semaine d'Histoire du Canada [... 1925 ...], 357.

73 Abbé Auguste Gosselin, Jacques Labrie, 128. 
1838 environ, les mots de Philippe Aubert de Gaspé, écolier du Séminaire en 1800, semblent encore d'actualité:

Confus d'ignorer l'histoire des Assyriens, des Mèdes et des Perses, celle de notre pays était jadis lettre morte pour nous. ${ }^{74}$

Des innovateurs comme l'abbé Painchaud tenteront de réagir contre cet état de choses. Dans le prospectus du collège qu'il fondera en 1829, il souligne l'importance de l'histoire nationale qu'il faut étudier avant toute autre. ${ }^{75}$ Et à Nicolet, à la même époque, un préfet des études et professeur qui illustrera plus tard l'historiographie canadienne-française entreprend d'intéresser ses élèves à l'histoire de notre pays. Gérin-Lajoie, le plus brillant de ces derniers, racontera plus tard avec émotion l'enthousiasme suscité par les leçons familières de l'abbé Ferland. ${ }^{76}$

Au Petit Séminaire de Québec, la première mention d'un enseignement de l'histoire du Canada comme matière distincte est de 1838-39.77 Et encore s'agit-il du Régime français seulement. Les cours donnés à cette époque se terminent comme celui-ci:

une Providence dont les desseins sont impénétrables aux humains voulait que plus tard, à la faveur des ténèbres de la nuit, un rival digne de Montcalm franchit ce précipice (que personne ne pensait à garder) pour venir chercher sur les plaines d'Abraham la mort et la victoire. ${ }^{78}$

Il reste difficile de préciser les débuts de l'enseignement de l'histoire nationale d'après 1760 . Ce serait entre 1843 et

74 Philippe Aubert de Gaspé, Les Anciens Canadiens, I: 229.

75 N.-E. Dionne, Vie de C'.-F. Painchaud, prêtre, curé, fondateur du collège de Sainte-Anne-de-la-Pocatière, 384. Le prospectus a été d'abord publié dans le Canadien du 1er août 1828.

${ }^{76}$ Biographie de l'abbé J.-B.-A. Ferland dans le Foyer Canadien, III (1865) : XXX.

77 Programme abrégé du cours d'étude du Petit Séminaire de Québec pour l'année 1838-39. Les Archives du Séminaire conservent un document curieux intitulé postérieurement: Embryon d'un cours d'histoire du Canada. Sommaire qui remonte $\grave{a}$ la domination française. ASQ, Polygraphie, 19. Rien n'autorise à croire à partir de cette pièce isolée qu'on aurait enseigné l'histoire nationale au Séminaire avant 1838.

78 ASQ, M-53, 172s. Cours donné en 1842-43 par l'abbé Holmes probablement. 
1853.79 Il semble que durant cette période et jusqu'à la parution de l'Histoire $d u$ Canada de l'abbé Laverdière en 1869 on n'ait pas fait usage de manuel. Certes, il existait sur le sujet ceux de Perrault publiés entre 1831 et 1836 mais rien ne nous permet de croire qu'ils aient servi au Séminaire. ${ }^{80}$

Ainsi, l'histoire nationale pénètre tardivement au Petit Séminaire et timidement au début. Ajoutons qu'à aucun moment son enseignement ne donnera lieu à des incidents comme celui de Saint-Hyacinthe en 1833 où peu s'en fallut qu'un examen d'histoire du Canada ne tournât mal. En effet, les élèves, en présence du gouverneur lui-même et à un moment de tensions politiques et raciales profondes, s'attachèrent à insister, dans leurs réponses orales, sur les défaites britanniques aux mains des Canadiens. Le scandale eut même des échos dans la presse du temps. ${ }^{81}$ Un tout autre esprit semble alors souffler sur l'enseignement dispensé au Petit Séminaire.

\section{(à suivre)}

Pierre SAVARD,
assistant à la Faculté des Arts (Québec)

79 En 1843, l'abbé Holmes dans son célèbre discours de fin d'année nous dit que les élèves de Seconde ont étudié cette année-là « l'histoire du Canada jusqu'à la conquête », Joseph-Edmond Roy, Souvenirs d'une Classe au Séminaire de Québec (1867-1877), 158. Un cahier de notes d'élèves nous apprend que l'abbé Taschereau enseigne en 1853-54 l'histoire du Canada « sous la domination anglaise », ASQ, M-94.

80 C'est l'opinion de l'abbé Maheux, Notes historiques sur le cours classique $[\ldots], 17$.

81 Chanoine C.-P. Choquette, Histoire du Séminaire de Saint-Hyacinthe depuis sa fondation jusqu'à nos jours, I: 158. 